\title{
PASV: Automatic protein partitioning and validation using conserved residues
}

\author{
Ryan M. Moore ${ }^{1}$, Amelia O. Harrison ${ }^{2}$, Daniel J. Nasko ${ }^{1}$, Jessica Chopyk ${ }^{3}$, \\ Metehan Cebeci $^{6}$, Barbra D. Ferrell ${ }^{2,4}$, Shawn W. Polson ${ }^{1,4,5}$ and K. Eric \\ Wommack ${ }^{2,4}$,*
}

${ }^{1}$ Center for Bioinformatics \& Computational Biology, University of Delaware, Newark, DE, USA and

${ }^{2}$ Department of Plant and Soil Science, University of Delaware, Newark, DE, USA and ${ }^{3}$ Department of Pathology, University of California San Diego, La Jolla, CA and 4Delaware Biotechnology Institute, Newark, DE, USA and ${ }^{5}$ Department of Computer and Information Sciences, University of Delaware, Newark, DE, USA and

${ }^{6}$ Department of Biological Sciences, University of Delaware, Newark, DE, USA

*wommack@udel.edu

\section{Abstract}

Background. Increasingly, researchers use protein-coding genes from targeted PCR amplification or direct metagenomic sequencing in community and population ecology. Analysis of protein-coding genes presents different challenges from those encountered in traditional SSU rRNA studies. Most protein-coding sequences are annotated based on homology to other computationally-annotated sequences, which can lead to inaccurate annotations. Therefore, the results of sensitive homology searches must be validated to remove false-positives and assess functionality. Multiple lines of in silico evidence can be gathered by examining conserved domains and residues identified through biochemical investigations. However, manually validating sequences in this way can be time consuming and error prone, especially in large environmental studies.

Results. An automated pipeline for protein active site validation (PASV) was developed to improve validation and partitioning accuracy for protein-coding sequences, combining multiple sequence alignment with expert domain knowledge. PASV was tested using commonly misannotated proteins: ribonucleotide reductase (RNR), alternative oxidase (AOX), and plastid terminal oxidase (PTOX). PASV partitioned 9,906 putative Class I alpha and Class II RNR sequences from bycatch in a global viral metagenomic investigation with $>99 \%$ true positive and true negative rates. PASV predicted the class of 2,579 RNR sequences in $>98 \%$ agreement with manual annotations. PASV correctly partitioned all 336 tested AOX and PTOX sequences.

Conclusions. PASV provides an automated and accurate way to address post-homology search validation and partitioning of protein-coding marker genes. Source code is released under the MIT license and is found with documentation and usage examples on GitHub at https://github.com/mooreryan/pasv.

Key words: active sites; bioinformatics; marker genes; metagenome; phylogenetic analysis; proteins; ribonucleotide reductase; software; validation; virome 


\section{Background}

Next generation DNA sequencing has continued to yield ever larger sequence datasets, enabling researchers to leverage vast amounts of sequence data in addressing a variety of scientific questions from cataloguing variation in human genomes [1] and connecting the gut microbiome with human health [2] to examining the circadian clock in soybean [3] and surveying viruses of the global ocean [4]. For example, sequencing has led to substantial advancements in understanding the community and population biology of microorganisms in nature. Nevertheless, while generation of data continually improves, accurate and comprehensive data analysis remains a challenge for investigations leveraging large sequence datasets.

Building on the example of microbial ecology, for decades researchers have relied on sequence based surveys of stable RNA genes, such as SSU rRNA, as phylogenetic markers for assessing the composition of cellular microbial communities. However, the focus on stable and highly conserved RNA gene sequences for microbial ecology studies has limited researcher's ability for fine scale delineation of cellular microbial populations from one another $[5,6]$ and identification of viral populations which do not encode SSU rRNA genes [7]. Use of protein-coding gene sequences as phylogenetic markers for community and population ecology studies can address these shortcomings of SSU rRNA analyses. However, accurate identification of protein-coding genes from either targeted amplicon libraries or shotgun metagenomes remains a significant analytical challenge.

In microbial ecology investigations, both stable rRNA and protein coding marker gene sequences are obtained either through targeted PCR amplification or direct sequencing (i.e., shotgun metagenome sequencing) of environmental DNA. Either approach has limitations that are addressed by the other. Targeted PCR amplification can deeply sample microbial populations within a community, detecting even the rarest of members; however, this approach may miss novel diversity by relying on previously sequenced genes for constructing PCR primers $[8,9,10]$. While every effort is made to ensure marker gene primers capture as much diversity as possible, amplification bias is always present [11]. In contrast, metagenome sequence libraries from shotgun sequencing provide a relatively unbiased picture of microbial diversity, with the caveat of a more limited ability for sampling rare populations $[12,13]$. With sequence assembly, this approach also provides the genomic context of marker genes, highly useful information for genome to phenome investigations [14]. Nevertheless, shotgun metagenomics presents significant additional analytical and computational requirements making this approach more expensive and difficult $[15,16]$. Furthermore, researchers still must drill down to the level of specific genes within metagenomes, such as those that have undergone extensive biochemical characterization, to uncover interesting biological and ecological patterns from the sequencing data $[17,18,19,20]$. In the case of either approach, accurately determining the identity of a sequence is critical in preventing subsequent errors in phylogenetic and functional analyses.

Assessing the potential gene functions within a community requires annotation of peptide sequences within metagenomes. Homology-based search tools such as BLAST [21] are the bedrock of sequence annotation, however, functional annotation of pro- teins based on homology can be error prone [22, 23]. Biochemically annotated proteins are relatively rare in major databases, and usually arise from studies of a few select model organisms $[24,25,26]$. As a result, many environmental sequences are annotated based solely on homology to other computationally annotated environmental sequences rather than to biochemically characterized proteins. Often, such environmental sequence annotations are several steps away from a confident, biochemical annotation, which can quickly lead to inaccuracies resulting from "error percolation" [24].

Furthermore, highly sensitive homology search tools used for annotating and identifying marker genes within metagenomes often have high false positive rates [27]. Identifying false positives in functional annotations is an active area of research and many techniques are available. Machine learning algorithms have been used for identifying false positives based on characteristics of multiple sequence alignments (MSAs) [28, 29]. Active site profiling, or examining the characteristics of regions close to a protein's active sites, has been used for sensitive and functionally relevant annotations [30, 31, 32, 33].

Even with accurate functional annotations, researchers need a means for predicting if a peptide sequence represents a functional enzyme. While a protein's function cannot be definitively determined in silico, evidence can be gathered by examining active sites, allosteric sites, and other key conserved residues established through biochemical investigations. However, manually validating key residues in thousands of peptide sequences using MSAs is time consuming, especially when considering the large volume of marker gene sequences obtained through amplicon or shotgun metagenome studies [4]. Furthermore, multiple sequence alignment quality degrades as the number of sequences in an alignment increases [34], or when the sequences to be aligned are highly divergent from one another [35].

To address the issue of accuracy in the validation of proteincoding gene sequences, an automated pipeline for protein active site validation (PASV) was developed. PASV provides researchers with a fast and accurate method for validating protein active sites and point mutations in particular genes of interest. Combining multiple sequence alignment with expert domain knowledge in an automated way, PASV more accurately identifies functional protein sequences within large sequence datasets. In this way, PASV can be used as a post-homology search processing step to eliminate most false positive hits and peptides that are likely to be non-functional. Additionally, PASV can be used to partition proteins into groups based on the residues present in functionally important positions of an alignment, such as conserved catalytic residues or residues with interesting biochemical properties (e.g., variants in motif B in DNA polymerase I [17]).

The accuracy of PASV was tested using commonly misannotated proteins: ribonucleotide reductase (RNR), alternative oxidase (AOX), and plastid terminal oxidase (PTOX) [36, 37]. In the first case, PASV was used to identify functional RNRs based on active site residues, and to differentiate Class I alpha and Class II RNRs based on a single amino acid residue. In the second case, PASV was used to distinguish two proteins commonly found in plants, AOX and PTOX, which have been previously shown to be difficult to differentiate with homology search alone, but can be readily partitioned using conserved residues [37]. 


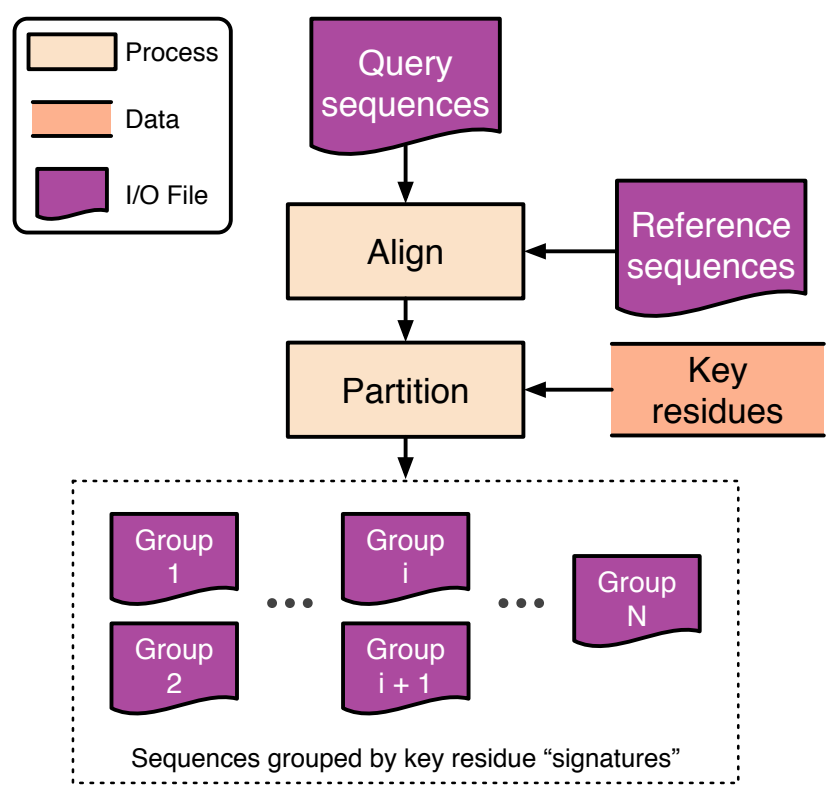

Figure 1. PASV conceptual diagram. PASV individually aligns each query sequence with a user-defined set of reference sequences. Then, columns of the resulting multiple sequence alignment are checked for user-defined key residue positions and, optionally, a region of interest (ROI). Finally, query sequences are partitioned into groups based on the amino acids at each of the key residues and whether the sequence spans the ROI.

\section{Methods}

\section{PASV Pipeline Overview}

PASV automates the process of aligning query sequences with a set of reference sequences and subsequently validating key residues and regions within the queries (Fig. 1). PASV is not a homology search tool. Rather it is a post-homology search filtering program. PASV uses a set of user-defined key amino acid residue positions to review alignment columns within multiple sequence alignments (MSAs). Key positions ideally will be residues that are both essential to the protein's function such as active sites and allosteric binding sites, and highly conserved across the diversity of known protein sequences. In this way, PASV leverages the user's domain knowledge for automated filtering and validation of functional proteins discovered through homology search. Alternatively, key positions may contain residues that, when mutated, display interesting biochemical properties. PASV automatically bins such amino acid variants, providing information on the functional diversity of a given protein. Finally, PASV can automatically filter out query sequences that fail to span a region of interest (ROI) on the reference sequences.

Prior to using PASV, users must select a set of reference sequences for the alignment. Special care should be taken when choosing a set of reference sequences, as picking an optimal reference set influences PASV's accuracy and runtime (see Results and Discussion sections for best practices). Reference sets are tailored to the protein of interest. That is, a set of references chosen for partitioning ribonucleotide reductase (RNR) sequences would not be the same as a set of references used to partition alternative oxidase (AOX) and plastoquinol terminal oxidase (PTOX). In addition to the reference set, which is developed once for a given protein of interest and then reused, the main input to PASV is a set of query protein sequences, generally obtained via a homology search for a protein of interest within a larger sequence dataset. PASV is especially useful in cases where there are many putative protein sequences to validate. For example, using a highly sensitive homology search tool (e.g., BLAST [21], HMMER [38], MMseqs2 [39], or PSI-BLAST [40]) against a metagenome often returns a large set of putative sequences that would be impractical for manual validation. PASV automates sequence validation avoiding time-consuming and potentially error-prone manual validation.

In the PASV pipeline, each query sequence is individually aligned with the reference sequences. PASV abstracts the process of aligning queries with references and identifying residues present in specific columns. Rather than reimplementing MSA algorithms, PASV leverages existing MSA software for aligning queries and reference sequences. It has built-in support for Clustal Omega [41] and MAFFT [42], but other alignment software can be specified at the command line by providing a custom specification.

For each alignment, PASV checks the residues of the query sequence aligning with the user-provided key residue positions in the reference set. The provided key residue positions are interpreted with respect to the original, unaligned first reference sequence. Each query is assigned a key residue "signature" based on these residues. PASV also optionally checks whether each query sequence spans a user-defined region of interest with respect to the reference sequences. Thus, PASV groups query sequences based on the key residue signature, and optionally by ROI spanning status. For example, in the case of RNR, the user may select key residue positions $437,438,439,441$, and 462 with respect to the first reference sequence. Then queries will be binned according to the residues that align with the reference sequences at those positions, i.e., their key residue "signatures" (Fig. 2).

\section{Implementation \& source code availability}

The PASV pipeline is implemented in Ruby (https://www. ruby-lang.org/), a dynamic, open source programming language. PASV leverages existing multiple sequence alignment software, such as Clustal Omega [41] or MAFFT [42], thus, a multiple sequence alignment program should be installed prior to running PASV. PASV is open-source software (MIT license) and is freely available on GitHub (https://github.com/mooreryan/ pasv). Rather than install PASV and its dependencies directly, a Docker image (https://hub.docker.com/r/mooreryan/pasv) and wrapper script (https://github.com/mooreryan/pasv/blob/master/ bin/pasv_docker) are also available. PASV v1.3.0 (https://github. com/mooreryan/pasv/releases/tag/v1.3.0) was used for all experiments.

\section{PASV result network diagrams}

Resulting PASV output files were converted to a node-link network diagram with a custom script (available on the PASV GitHub page) and visualized with Cytoscape v3.7.1 [43].

\section{Collecting RNR sequences}

\section{Retrieving RNR sequences from the RNRdb}

All available Class I alpha and Class II RNRs were retrieved from the RNRdb on August 20, 2018 [36]. These 66,209 RNR peptide sequences were dereplicated (exact and substring matches) using CD-HIT v4.6 [44], yielding 29,401 representative sequences. Sequences were then divided into closely related groups (clades) 


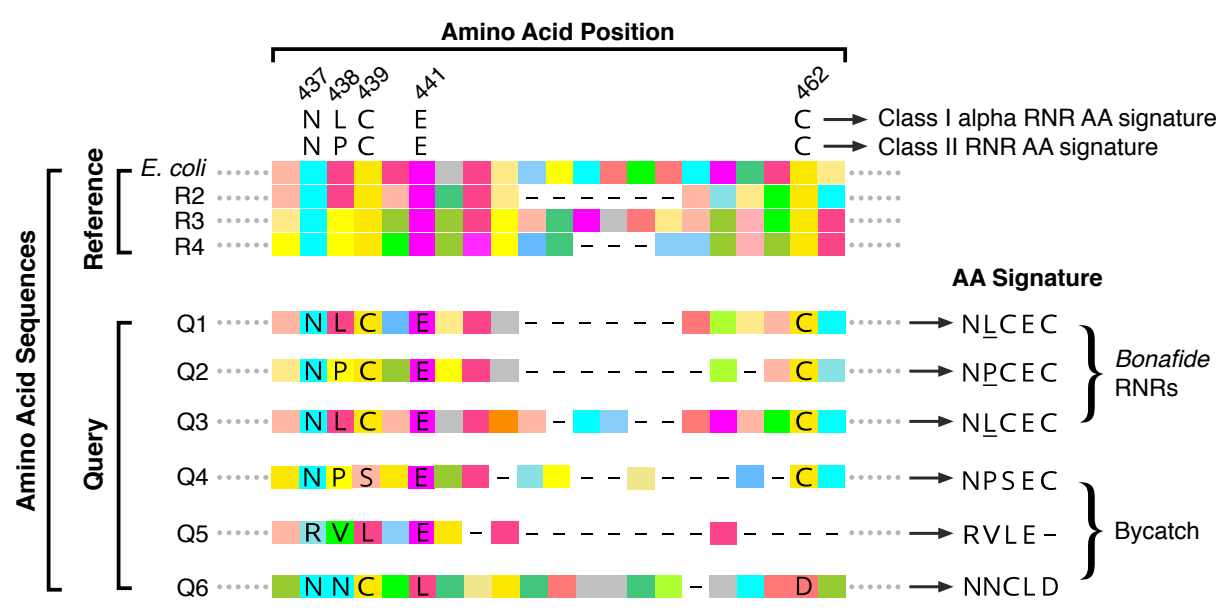

Figure 2. RNR classification and partitioning example. PASV aligns each query sequence individually with all reference sequences (in this case, four references). Labelled positions are the user-specified key residues. The coordinates are specified with respect to the original positions on the unaligned first reference sequence (here, E. coli). Each query is assigned a signature based on the residues that align in the same columns as the key residues. In the case of RNR, residues N437, C439, E441, and C462 are required, while residue 438 is diagnostic of RNR class (L438 indicates Class I alpha and P438 indicates Class II). In this example, queries 1, 2, and 3 have NCEC in the correct positions and are considered to be bonafide RNRs. Queries 1 and 3 can be classified as Class I alpha based on L438, whereas query 2 can be classified as Class II based on P438. Queries 4, 5, and 6, do not have the required NCEC signature and are thus considered bycatch.

as defined by the RNRdb for manual assessment of active site residues and intein removal [23]. From the 29,401 representative sequences, 286 sequences were removed as they lacked one or more of the four residues essential for RNR function (N437, C439, E441, C462 with respect to Escherichia coli K12 W3110 ribonucleoside diphosphate reductase 1 alpha subunit, accession no. WP_001075164.1) [45, 46, 47, 48]. The 29,133 remaining RNRs were retained for downstream analysis.

\section{RNRdb sequence tree \& phylogenetic clustering}

To reduce the number of sequences used for building a phylogenetic tree of known RNR peptides, the 29,133 bonafide RNRdb sequences were clustered with MMseqs2 (version e1a1c1226ef22ac3doda8e8f71adb8fd2388a249) [39] at 75\% identity over $80 \%$ of the alignment length, resulting in a set of 2,579 peptide clusters. Cluster centroids were aligned with MAFFT v7.427 using the FFT-NS-2 method [42]. Columns of the resulting multiple sequence alignment containing $>95 \%$ gaps were removed. Finally, FastTree v2.1.10 with double precision arithmetic [50] was used to build the tree, and the resulting tree was midpoint-rooted with a custom Python script (https://github. com/mooreryan/midpoint-root) using ETE Toolkit v3 [51]. Different numbers of phylogenetic RNR clusters were generated by collapsing branches whose lengths were below a threshold using iTOL [49]. Six different clustering scenarios were used representing six levels of phylogenetic granularity (4 clusters: collapsed branch length (BRL) < 3.75; 8 clusters: BRL < 3.1; 14 clusters: $\mathrm{BRL}<2.85 ; 19$ clusters: $\mathrm{BRL}<2.65 ; 24$ clusters: 2.485 ; and 29 clusters: BRL < 2.34) (Fig. 3).

\section{Retrieving RNR sequences from the Global Ocean Viromes dataset} The 1,995,784 Global Oceans Virome (GOV) [52] peptides (downloaded from: https://datacommons.cyverse.org/browse/iplant/ home/shared/iVirus/GOV/Contigs_set, file last modified 2017-0423) were searched against RNRdb sequences with MMseqs2 (sensitivity: 7, max-seqs: 1000, num-iterations: 3, start-sens: 1 , sens-steps: 7, default e-value cutoff: 0.001 , defaults for all other options). This search yielded 12,412 virome sequences. Sequences having fewer than 100 amino acids were removed, leaving 9,906 sequences. These sequences were manually curated using a combination of conserved residues, domains, and phylogenetic placement (as in [23]) resulting in 2,916 bonafide RNRs and 6,990 non-RNRs.

\section{Reference sets and PASV accuracy}

\section{Full reference set test}

Given that PASV uses MSA for validating key residues, PASV's accuracy is dependent on the chosen reference set and aligner. An experiment testing 1,920 combinations of reference sets, query sets, and aligners was used to determine those variables most affecting accuracy (Fig. 4). First, randomly selected reference sequence sets were compared to sets where selection was guided by a phylogenetic tree. For phylogenetically selected references, a tree containing 2,579 RNR sequences was partitioned at six levels of granularity $(4,8,14,19,24$, and 29 clusters (Fig. 3)). Two approaches were then taken for phylogenetic reference selection. First, phylogenetic reference sets were generated by selecting a single reference sequence from each tree clade (clades defined by various minimum branch lengths (BRL, Fig. 3) to test whether increasing the evenness of representation among rarer or divergent clades would improve PASV accuracy. Second, phylogenetic reference sets were generated by weighting the selection of sequences according to the number of sequences within a cluster (one reference sequence for every 200 sequences in the cluster) (Fig. 3). For each of the phylogenetically selected reference sets (including weighted and unweighted at all six levels of granularity), sizematched, randomly selected reference sets were included as controls. Finally, for each reference set selection criteria (phylogenetic or random, single or multi, reference set size), ten replicates were generated. Each reference set was tested with two aligners, MAFFT v7.427 [42] and Clustal Omega v1.2.4 [41], and two different query sets (RNRdb queries: 100 bonafide RNRs and 100 invalid RNRs missing key functional residues; Global Ocean Virome (GOV) queries: 200 bonafide RNRs and 100 invalid RNRs missing key functional residues). All experiments were run on an Intel(R) Xeon(R) CPU E5-2695 v4 @ 2.10GHz server with 36 
bioRxiv preprint doi: https://doi.org/10.1101/2021.01.20.427478; this version posted February 2, 2021. The copyright holder for this preprint (which was not certified by peer review) is the author/funder, who has granted bioRxiv a license to display the preprint in perpetuity. It is made available under aCC-BY 4.0 International license.

Moore et al. | 5

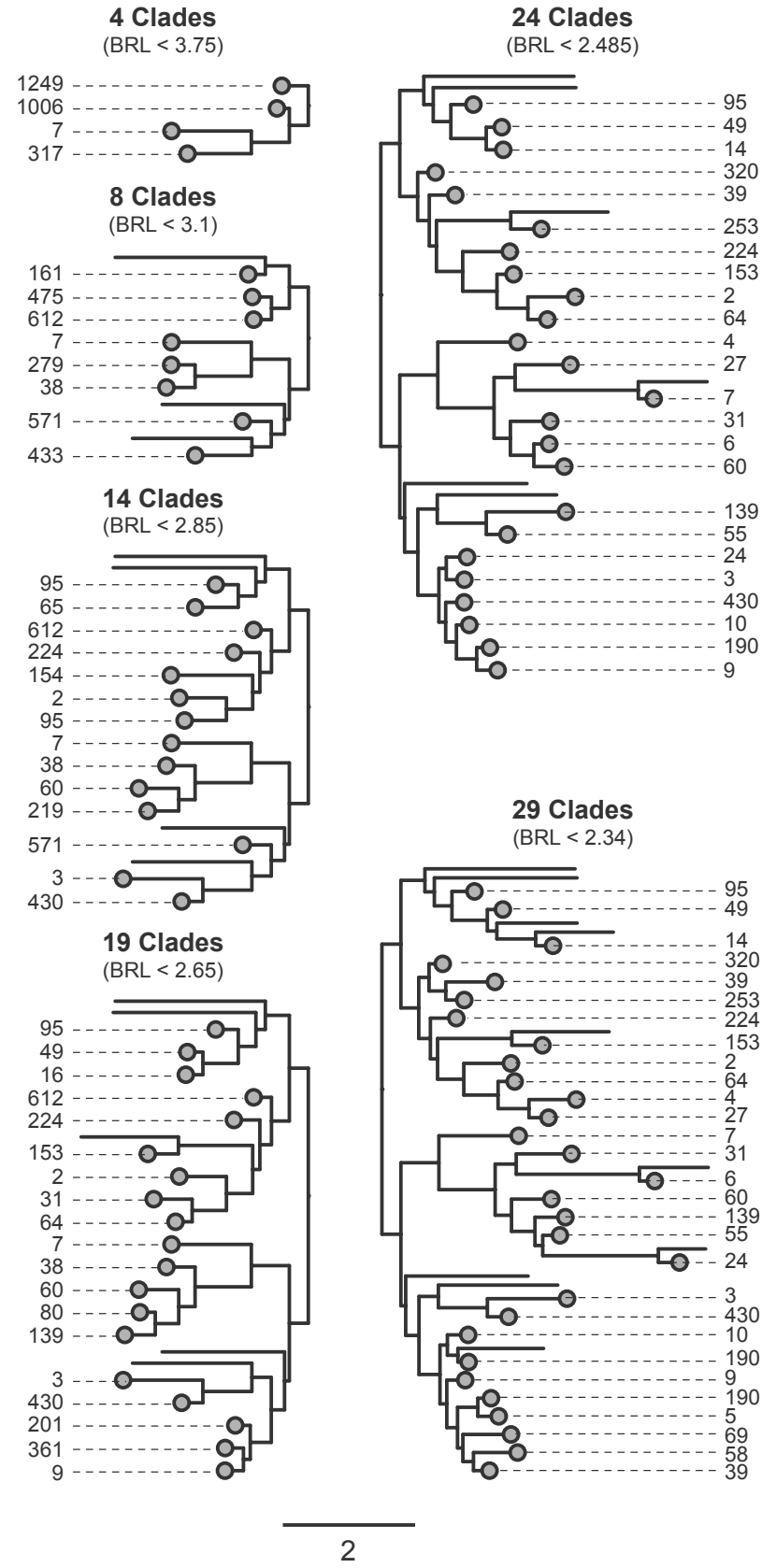

Figure 3. Phylogenetic clustering of ribonucleotide reductase proteins. Ribonucleotide reductases (RNRs) from the RNRdb [36] were clustered with MMseqs2 [39] at $75 \%$ identity over $80 \%$ of the alignment length. Phylogenetic clusters (grey circles) were created in iTOL [49] by collapsing clades with branch lengths (BRL) less than the amount shown. Leaf labels show the number of sequences within the clade. Branches without grey dots represent singleton clusters, and were not included in the pool of potential reference sequences. Scale bar represents amino acid substitutions per site.

cores ( 2 threads per core) and $512 \mathrm{~GB}$ of ram, with PASV set to use 68 threads (i.e., process 68 queries concurrently).

In summary, a total of 1,920 experiments were conducted. Six levels of phylogenetic tree partitioning were used (6 reference sets) (Fig. 3), each generating either a single (unweighted) or multiple (weighted) reference sequences per clade $(6 * 2=12$ reference sets). For each of these twelve groups, ten replicates

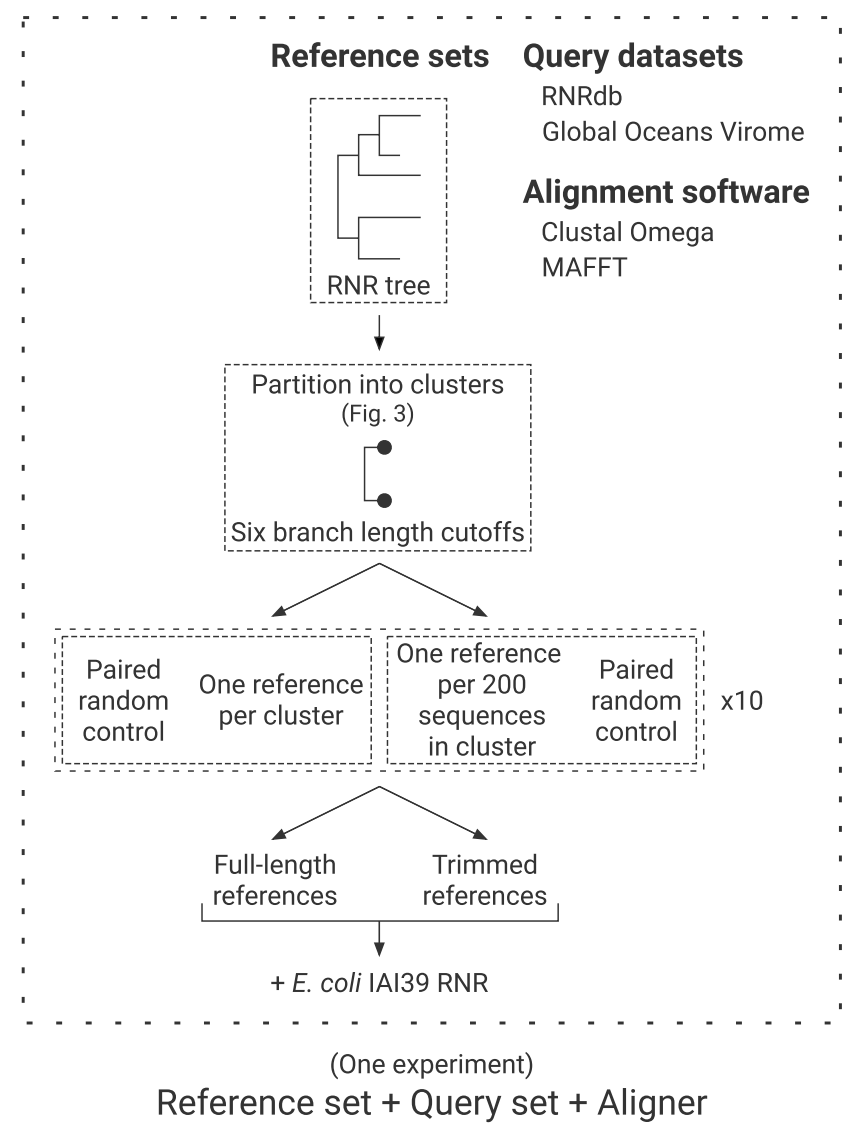

Figure 4. PASV reference set test. Conceptual diagram of the validation experiment testing the effects of reference set, query set, and aligner on PASV accuracy. One experiment is a PASV run with a unique combination of a reference set, a query set, and an aligner. The reference sequence selection strategy (phylogenetically-guided or random), the size of the reference set (numbers of sequences and their distribution across the known diversity of a protein), and the length of reference sequences (full length or smaller region of interest) were tested for their impact on PASV accuracy in correctly identifying manually curated sequences. For each reference set category, 10 random samples (i.e., replicates) were generated. For each reference set, two aligners (Clustal Omega [41], and MAFFT [42]), and two query sets (RNRdb [36] and Global Ocean Virome (GOV) [52]) were run.

were made $(12 * 10=120$ reference sets $)$. For each of these 120 reference sets, size-matched, randomly selected reference sets were used as controls $(120 * 2=240$ reference sets $)$. For each of these 240 reference sets, both full-length reference sequences, and reference sequences trimmed to the shorter region of interest (ROI, positions $437-605$, E. coli numbering) were tested $(240 *$ $2=480$ reference sets). For each of these 480 reference sets, two aligners (Clustal Omega or MAFFT) were tested $(480 * 2=960$ reference sets + aligners). For each of these 960 reference set plus aligner pairs, two different query sets (RNRdb or GOV) were tested $(960 * 2=1,920$ experiments) (Fig. 4).

\section{Putative GOV RNR queries test}

GOV RNR sequences (9,906 sequences) were used to test PASV on a dataset more reflective of an actual use case. Because most of the variables tested in the full reference set test had little effect on PASV accuracy (see Results), and due to the size of the query set, a reduced set of variables was used to generate reference sets. References from three clustering levels $(8,19,29)$ with 
both phylogenetic and random sequence picking were generated in triplicate, yielding 18 reference sets. For the other variables included in the full reference set test, only the top performing options were used in this experiment: Clustal Omega rather than MAFFT, full-length references rather than trimmed, and one sequence per clade vs one sequence for every 200 sequences per clade. All experiments were run on the same server as the full reference set test with PASV set to run 68 concurrent alignment jobs.

\section{Data analysis}

Data analysis was performed in R v3.6.3 [53] with tidyverse v1.3.0 [54] and ggplot2 v3.3.0 [55]. All true positive and true negative rate linear models were calculated with the $1 \mathrm{~m}$ function in $\mathrm{R}$. Model coefficients were considered significant if their $p$-values were less than 0.05 as reported by the $\mathrm{R}$ function summary. $1 \mathrm{~m}$. All box and whisker plots were made using the geom_boxplot function from ggplot2. All scatter plot regression lines were made using the geom_smooth function from ggplot2 using locally estimated scatterplot smoothing (LOESS, default parameters) with 95\% confidence intervals, except for Additional Files 1 and 4 which use linear regression with $95 \%$ confidence intervals calculated with geom_smooth using $1 \mathrm{~m}$. All point jittering was done using the geom_jitterdodge function from ggplot2.

\section{Analyzing putative and bonafide GOV RNRs}

\section{GOV RNR trees}

The 9,906 putative RNR sequences identified through homology search alone, and the 2,914 PASV-predicted bonafide RNR sequences (using the reference set chosen from the best practices according to the full reference set test and the GOV RNR queries test) were aligned with MAFFT v7.427 FFT-NS-2 [42]. Columns with $>95 \%$ gaps were removed and a phylogenetic tree was inferred with FastTree v2.1.10 double precision arithmetic [50]. The resulting Newick tree files were visualized with Iroki [56].

\section{Annotating GOV tree sequences}

Sequences were manually selected from clades containing only non-RNRs (according to manual curation) from the phylogenetic tree containing all 9,906 putative RNRs from GOV. Sequences were searched against National Center for Biotechnology Information's Conserved Domain Database (NCBI CDD) v3.18 and the top domain hit by e-value was recorded [57]. All sequences that had a mismatch between manual curation and PASV prediction in any of the 18 full GOV experiments were also searched against the conserved domain database using Batch CD-Search [58] and the top domain hit was recorded. In the case that multiple domains were identified, the top hit was recorded for each domain (Additional File 10).

\section{Partitioning RNR classes}

To test PASV's ability to partition Class I RNR alpha subunit sequences from Class II RNR sequences, the 2,579 clusters from the RNRdb tree (Fig. 3) were used as PASV query sequences with the "best practices" RNR reference set. In addition to the same N437, C439, E441, and C462 key residues (E. coli numbering) used in previous experiments, residue L/P438 was also included. Any sequence PASV identified as having NLCEC was labeled as a Class I alpha RNR, whereas any sequence with NPCEC was classified as a Class II RNR. Any sequences with key residue signatures other than the NLCEC for Class I alpha and NPCEC for Class II were grouped into the "Other" category. The PASV predictions were compared with RNRdb assigned class annotations.

\section{Partitioning AOX and PTOX}

Alternative oxidase (AOX) and plastid terminal oxidase (PTOX) peptide sequences were collected from a recent study [37]. Sequences from supplemental data sheet 1 , containing 14 fulllength PTOX proteins that were previously erroneously annotated as AOX, and sequences from supplemental data sheet 2, representing trimmed AOX and PTOX sequences, were obtained. Some of the trimmed sequences in supplemental data sheet 2 had accession numbers with which the corresponding full length sequences could be recovered from NCBI databases using the Entrez Direct efetch [59]. Forty-eight full-length AOX and eight PTOX sequences were recovered in this manner. Recovered full length sequences were combined with trimmed sequences yielding a set of 336 query sequences for PASV testing.

The ability of PASV to classify both AOX and PTOX sequences within a mixed set of peptide sequences was tested with two separate PASV runs: once with an AOX reference set (UniProt entry IDs 022048,022049 , and E1CIY3; sequences selected from those manually annotated as AOX in [37]) and once with a PTOX reference set (UniProt entry IDs A0A061GHF5, B9RXE2, and Q56X52; sequences selected from those manually annotated as PTOX in [37]) sequences. In the AOX run, all query sequences were checked for conserved residues from AOX motifs 1 (E233, R234, M235, H236, L237, M238, T239) and 2 (L283, E284, E285, E286, $\mathrm{A} 287$ ), and sequences containing the correct residues were labeled as AOX, while sequences with other residues at these positions were labeled as non-AOX (numbering with respect to sequence 022048) [37]. For the PTOX run, all queries were checked for conserved residues from PTOX motifs 1 (G157, W158, R160, R161) and 2 (H177, H178, L179, L180, M182, E183), and any sequences containing the correct residues were labeled as being PTOX, while sequences with other residues at these positions were labeled as non-PTOX (numbering with respect to sequence AoA061GHF5) [37]. Finally, the sequence labels from the AOX and the PTOX run were combined for the final classification. Two positions were excluded from the motifs that were presented in [37] (159 in motif 1 , and 181 in motif 2 ) as these positions were more variable than the other motif positions.

\section{Results}

\section{What factors influence PASV accuracy?}

True positive and true negative rates for PASV validated RNR peptide sequences were explored with linear models. For GOV query sequences, aligner and reference trimming had a significant ( $p$-value $<0.05$ ) association with both true positive and true negative rates (Fig. 5). Clustal Omega was associated with an $11.1 \%$ increase in true positive rate and a $0.2 \%$ decrease in true negative rate as compared to MAFFT. Full length references had a $12.6 \%$ increase in true positive rate and a $0.07 \%$ increase in true negative rate as compared to references trimmed to the region of interest. While statistically significant according to the linear model, variables associated with true negative rate had negligible effect in practice for GOV queries. For RNRdb queries, when all variables were included as predictors, aligner and reference trimming were both significant predictors of true positive 


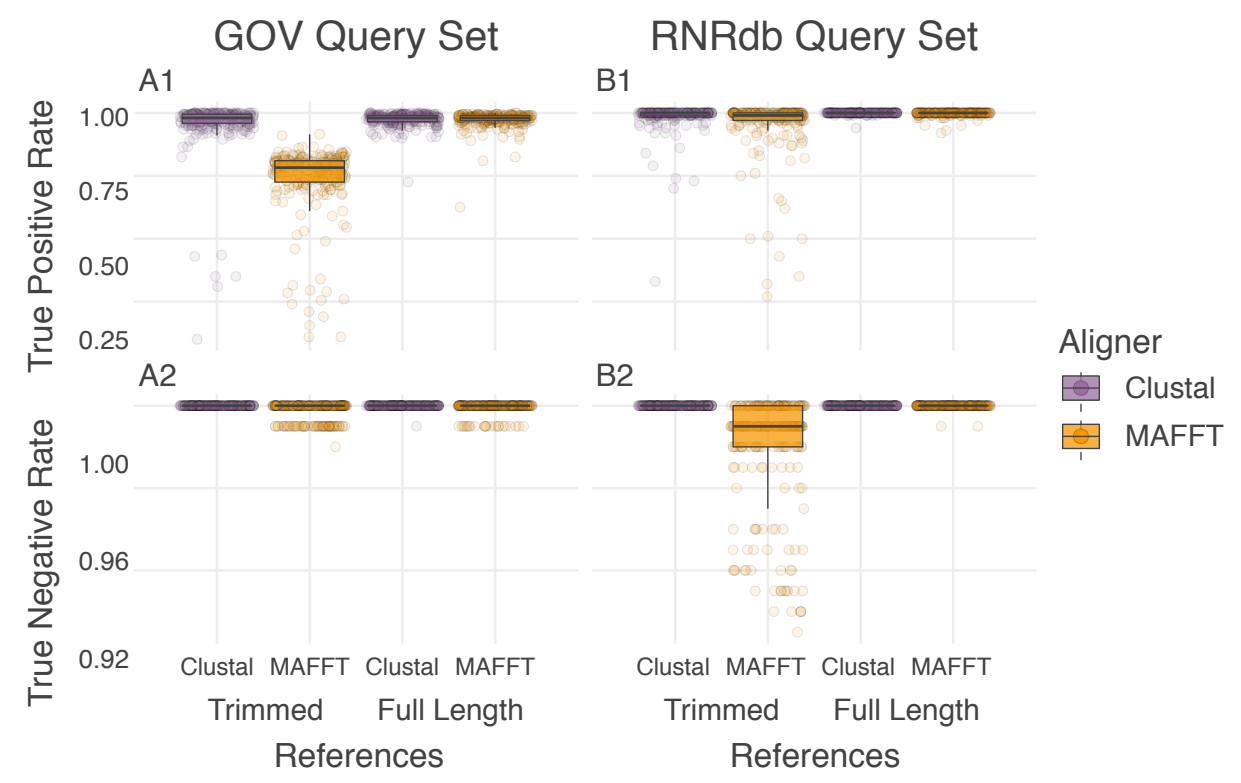

Figure 5. PASV accuracy is influenced by aligner and reference trimming. PASV true positive (A1 \& B1) and true negative rates (A2 \& B2) across reference sets of RNR peptide sequences. Results are shown for the Global Ocean Virome (GOV) query set (A) and the RNRdb query set (B). Each dot represents a single PASV run (i.e., one reference set with an aligner). Box (showing median and interquartile range (IQR)) and whisker (1.5 x IQR) plots are overlaid. Within each panel, PASV tests are partitioned by reference sequence length (full length references vs. those trimmed to the region of interest) and by multiple sequence aligner (Clustal Omega - purple vs. MAFFT - orange).

rate. Clustal Omega was associated with a 1.5\% increase, and full length references were associated with $2.7 \%$ increase in true positive rate. For true negative rate, all variables other than replicate were significant; however, all effects were quite small $(<1.3 \%)$.

Given that full-length references were superior to those trimmed to a ROI (Fig. 5), only full-length references were included in subsequent analysis of covariate effects on PASV accuracy. Full-length reference sets split into groups based on query set (GOV vs RNRdb) and aligner (Clustal Omega vs. MAFFT) were re-run through linear models on the following five remaining covariates: (1) number of tree clusters; (2) number of reference sequences; (3) single or multiple reference sequences chosen per clade (single/multi); (4) random or phylogenetically-guided reference sequence choice (random/phylo); and (5) reference set replicate (Table 1).

The PASV true positive rate decreased with the number of tree clusters used in the phylogenetically-guided reference sequence choice approach (Fig. 3), but increased with respect to the number of references for GOV-MAFFT, RNRdb-MAFFT, and RNRdbClustal groups (Table 1). When using MAFFT, picking a single reference from each clade as opposed to weighting the number of references by number of sequences in the clade was associated with a significantly higher true positive rate for both GOV and RNRdb query sets; however, this trend was not seen when using Clustal (Table 1). Overall, choosing references randomly (when using MAFFT, but not Clustal) and including more sequences in the reference set were associated with better PASV accuracy. However, the positive effect of the number of reference sequences on true positive rate plateaued after ca. 20 reference sequences (Fig. 6). Additionally, the effect of increasing the number of references is more pronounced with the MAFFT aligner than with Clustal Omega (Fig. 6).

While using an increasing number of references boosted PASV accuracy, it also increased runtime (Additional File 1), as more sequences needed to be aligned. Using full-length references as opposed to references trimmed to the region of interest also increased the runtime. This is due to full-length references containing more bases that need to be aligned. Another consideration for run-time is the alignment algorithm: running PASV with Clustal Omega was faster than with MAFFT (Additional File 1).

In summary, variables that had the most impact on PASV true positive and true negative rate were alignment software (with Clustal Omega outperforming MAFFT) and reference trimming (full-length references performing better than references trimmed to the ROI) (Fig. 5, Table 1, Additional File 2).

\section{Testing PASV with the full GOV query set}

PASV was tested on a large metagenomic query set using best practices determined from the 1,920 reference set tests. The only variable significantly associated with PASV accuracy was phylogenetic vs. random reference picking, which affected the true negative rate; however, the size difference was small $(0.027 \%)$ (Additional File 3). As the different reference sets all had comparable results, the mean and $95 \% \mathrm{CI}$ of all 18 reference set runs was used for the confusion matrix. Overall, PASV was highly concordant with the manual curation, with $>99 \%$ agreement between PASV predictions and manual curation (Table 2). As in the full reference set tests, runtime increased with increasing numbers of reference sequences (Additional File 4) (linear model: runtime $=$ $(-13.5 \pm 6.8)+(5.26 \pm 0.3) *$ number of reference sequences $)$.

PASV provides a means for automating the process of validating the identity of peptide sequences collected through homology search. The algorithm partitions query peptides into bonafide and by-catch sequences (Fig. 1). Given this, the impact of including by-catch sequences in a phylogenetic analysis of metagenomic RNR sequences was examined. Phylogenetic trees of putative RNR sequences from GOV ( 9,906 sequences), and sequences 
bioRxiv preprint doi: https://doi.org/10.1101/2021.01.20.427478; this version posted February 2, 2021. The copyright holder for this preprint (which was not certified by peer review) is the author/funder, who has granted bioRxiv a license to display the preprint in perpetuity. It is made 8 | Moore et al. available under aCC-BY 4.0 International license.

Table 1. Linear model coefficients with $p$-value < 0.1 for PASV reference set test (full-length references only).

\begin{tabular}{|c|c|c|c|c|c|}
\hline \multirow{2}{*}{ Model } & \multirow{2}{*}{ Variable } & \multicolumn{4}{|c|}{ Coefficient \pm Standard Error } \\
\hline & & GOV-MAFFT & GOV-Clustal & RNRdb-MAFFT & RNRdb-Clustal \\
\hline \multirow{6}{*}{ True positive rate } & Intercept & $86.50 \pm 1.86$ & \multirow[t]{6}{*}{$97.60 \pm 1.69$} & $97.30 \pm 0.55$ & $99.29 \pm 0.28$ \\
\hline & No. tree clusters ${ }^{a}$ & $-0.56 \pm 0.13$ & & $-0.12 \pm 0.39$ & $-0.03 \pm 0.02$ \\
\hline & No. references ${ }^{\mathrm{b}}$ & $0.78 \pm 0.15$ & & $0.17 \pm 0.45$ & $0.05 \pm 0.02$ \\
\hline & Single vs. multi ${ }^{c}$ & $4.77 \pm 1.33$ & & $1.10 \pm 0.39$ & \\
\hline & Random vs. phylo ${ }^{\mathrm{d}}$ & $0.65 \pm 0.36$ & & $0.24 \pm 0.11$ & \\
\hline & Replicate & & & & \\
\hline \multirow{6}{*}{ True negative rate } & Intercept & $99.92 \pm 0.19$ & \multirow[t]{6}{*}{$99.99 \pm 0.43$} & \multirow[t]{2}{*}{$99.87 \pm 0.06$} & $100.00 \pm 0.00$ \\
\hline & No. tree clusters & & & & $0.00 \pm 0.00$ \\
\hline & No. references & & & \multirow[t]{4}{*}{$0.01 \pm 0.00$} & \multirow[t]{4}{*}{$0.00 \pm 0.00$} \\
\hline & Single vs. multi & & & & \\
\hline & Random vs. phylo & $-0.12 \pm 0.04$ & & & \\
\hline & Replicate & $-0.01 \pm 0.01$ & & & \\
\hline
\end{tabular}

${ }^{a}$ Number of tree clusters

${ }^{b}$ Number of reference sequences

${ }^{\mathrm{c}}$ Single or multiple reference sequences chosen per clade

$\mathrm{d}_{\text {Random or phylogentically-guided reference sequence selection }}$

Table 2. Confusion matrix of PASV results for 18 references sets against putative GOV RNR sequences.

\begin{tabular}{lrr}
\hline \multirow{2}{*}{ PASV Prediction } & \multicolumn{2}{c}{ Manual curation } \\
\cline { 2 - 3 } & \multicolumn{1}{c}{ Positive } & \multicolumn{1}{c}{ Negative } \\
\hline Positive & $2894.6 \pm 5.3$ & $12.5 \pm 0.8$ \\
Negative & $21.4 \pm 5.3$ & $6977.5 \pm 0.8$ \\
\hline
\end{tabular}

Mean $\pm 95 \%$ confidence interval for 18 PASV runs. Each run is one of 18 reference sets with the full 9,906 sequence Global Ocean Virome (GOV) query set.

from the putative RNRs that PASV identified as bonafide (i.e., those sequences with N437, C439, E441, C462, E. coli numbering) were compared (Additional File 5). For this PASV run, the best performing reference set (hereby referred to as the "best practices" reference set) of the 18 tested on the full GOV query set that also followed the best practices observed in the full reference set test (i.e., full-length, single sequence per clade, random selection) was used. This PASV run yielded 2,914 bonafide RNR sequences (i.e., those sequences with N437, C439, E441, C462, E. coli numbering).

The tree including all putative RNRs contained a high proportion of sequences on long branches, indicative of distantly related sequences or sequences with poor alignment (Fig. 7A). In contrast, the bonafide PASV sequence tree contained fewer long branches and more reasonable topology [60] (Fig. 7B). In the case of both trees, clades with long branches did contain nontarget sequences such as helicases, DNA polymerases, terminase, and thioredoxin (Fig. 7 and Additional File 6). However, the tree containing bonafide RNR sequences had substantially fewer long branches, and those that were present would be relatively easy to identify and remove. In practice, having fewer long branches reduces the time necessary for manual curation of phylogenetic trees.

Across all 18 GOV PASV runs (1 run per generated reference set), a total of 187 sequences out of 9,906 showed disagreement between PASV predictions and manual curation. These $187 \mathrm{se-}$ quences were annotated using NCBI CDD (Table 3, Additional File 3). Annotations of the 162 PASV predicted negative, manual $\mathrm{cu}-$
Table 3. NCBI CDD annotations of sequences with mismatched PASV prediction and manual curation.

\begin{tabular}{lrr}
\hline \multicolumn{1}{c}{ Annotation } & \multicolumn{2}{c}{ Count } \\
\cline { 2 - 3 } & $\begin{array}{c}\text { PASV positive, } \\
\text { manual curation } \\
\text { negative }\end{array}$ & $\begin{array}{c}\text { PASV negative, } \\
\text { manual curation } \\
\text { positive }\end{array}$ \\
\hline RNR (subclass unknown) & 5 & 96 \\
Class I RNR alpha subunit & $*$ & 3 \\
Class I RNR beta subunit & 2 & - \\
Class II RNR & 2 & 63 \\
Helicase & 4 & - \\
Pol I & 3 & - \\
Endonuclease & 2 & - \\
Terminase & 1 & - \\
Ankyrin repeat & 1 & - \\
No match & 1 & - \\
\hline
\end{tabular}

Counts are totals across 18 PASV runs: the full 9,906 sequence Global Ocean Virome query with 18 different reference sets.

${ }^{*}$ Sequences erroneously categorized as non-RNR by manual curation

ration positive sequences included three Class I RNR alpha subunits, 63 Class II RNRs, and 96 RNRs with unknown subclass. Sequences with hits to the RNR_PFL superfamily were considered to be either Class I alpha or Class II RNRs for two reasons: 1) other members of the supergroup, pyruvate formate lyase (PFL) and Class III RNRs, are oxygen-sensitive [61, 62], and thus unlikely to be found in the environments sampled in the GOV study [52]; and 2) these sequences grouped with other Class I alpha and Class II sequences on the phylogenetic trees (Fig. 7). The 25 remaining mismatched sequences (i.e., PASV predicted positive, manual curation negative) had more heterogeneous annotations. Twelve of these had hits to non-RNR domains: four helicases, three Pol Is, two endonucleases, one terminase, one Ankyrin repeat, and one with no match. Thirteen had hits to RNR domains: four Class I alpha subunit, two Class I beta subunit, two Class II RNR, and five RNRs with unknown subclass. The six sequences annotated as RNR Class I alpha subunits and Class II represent se- 


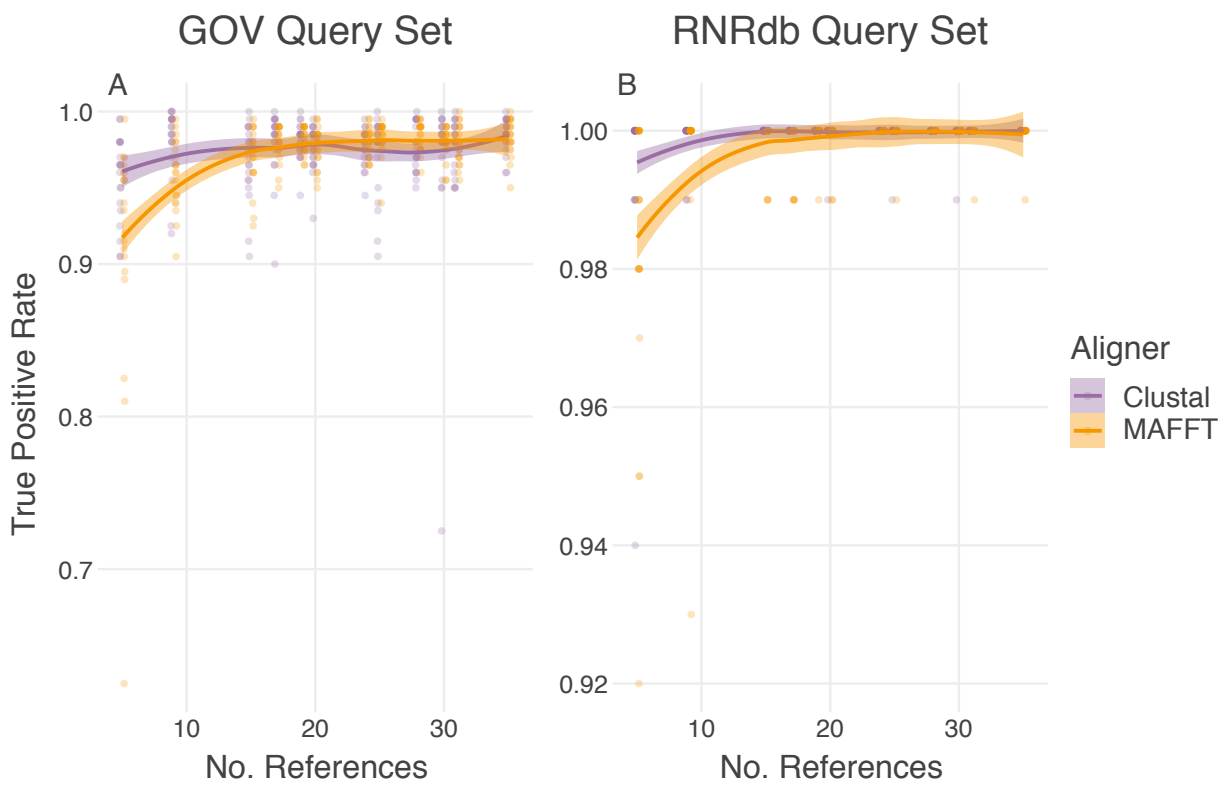

Figure 6. PASV true positive rate increases with number of references. Number of references per reference set versus PASV true positive rate for full-length reference sets. GOV query set and RNRdb query set are shown in panel A and panel B, respectively. Each dot represents a single PASV run (i.e., one reference set with an aligner: Clustal Omega - purple, MAFFT - orange). Locally estimated scatterplot smoothing (LOESS) lines with $95 \%$ confidence intervals are shown for each aligner. (Note the difference in $\mathrm{y}$-axis scale between panels A and B.)

Table 4. PASV Class I alpha and Class II predictions.

\begin{tabular}{lrr}
\hline \multirow{2}{*}{ PASV Prediction } & \multicolumn{2}{c}{ Manual annotation } \\
\cline { 2 - 3 } & Class I alpha & \multicolumn{1}{c}{ Class II } \\
\hline Class I alpha $^{\mathrm{a}}$ & $98.96 \%$ & $0.30 \%$ \\
Class II $^{\mathrm{b}}$ & $0.08 \%$ & $98.27 \%$ \\
Other $^{\mathrm{c}}$ & $0.96 \%$ & $1.43 \%$ \\
\hline
\end{tabular}

${ }^{\mathrm{a}}$ NCEC sequences with $\mathrm{L} 438$ (E. coli numbering)

${ }^{\mathrm{b}}$ NCEC sequences with $\mathrm{P} 438$ (E. coli numbering)

${ }^{\mathrm{c}}$ Non-NCEC sequences or those with any other residue at position 438

quences that were likely erroneously categorized during manual curation. Thus, PASV was correct according to CDD annotations in 175 of the 187 cases in which PASV predictions disagreed with manual annotation.

\section{Partitioning RNR Class I alpha subunit and Class II se- quences}

PASV's ability to partition two biochemical classes of RNR sequences (Class I alpha subunit and Class II [63, 62]) was examined. The 2,579 RNRdb sequences used to make the RNR tree for phylogenetic clustering (Fig. 3) were partitioned into Class I alpha subunits and Class II sequences using PASV. As the NCEC residues within the RNR PASV profile are required for RNR function $[45,46,47,48]$, any sequence without NCEC at residues $437,439,441$, and 462 (E. coli numbering) in the PASV run were grouped into the "Other" category. These included five Class I alpha and four Class II sequences. For the remaining 2,570 NCEC sequences, any sequence that PASV predicted as having an leucine at position 438 was labeled as a Class I alpha subunit, whereas any sequence with a proline at that position was predicted to be Class
II. These PASV predictions were compared to RNRdb annotations, and the results were recorded in Table 4 (Additional File 7). Of the 1,244 annotated Class I alpha sequences, PASV predicted 1,236 of them to be Class I alpha (correct PASV predictions: $98.96 \%$ ), one to be Class II (0.08\%), and seven to be "Other" (0.96\%). For the 1,326 annotated Class II sequences, PASV predicted 1,307 of them to be Class II (correct PASV predictions: 98.27\%), four to be Class I alphas (0.30\%), and 15 "Others" (1.43\%).

\section{Partitioning AOX and PTOX sequences}

A total of 336 alternative oxidase (AOX) and plastid terminal oxidase (PTOX) peptide sequences were recovered from a previous study examining misannotation of the AOX and PTOX gene groups in plants [37]. These sequences were classified with PASV using residues from the diagnostic, conserved motifs identified in [37]. This experiment tested the ability of PASV for correctly binning a mixed collection of AOX and PTOX peptide sequences. While distinct proteins, AOX and PTOX share regions of homology and are frequently missanotated by standard methods [37]. However, two motifs for each protein, when used in conjunction with MSA, enables correct classification of the proteins. Two reference sets were constructed, one to classify AOX and one to classify PTOX. The entire query set (336 total sequences, 254 AOX, 82 PTOX) was run through the PASV algorithm against both reference sets (Additional Files $8 \& 9$ ). In the AOX run, any sequence with the correct residues in the conserved motifs as identified by PASV was considered an AOX (motif 1: E233, R234, M235, H236, L237, M238, T239; motif 2: L283, E284, E285, E286, A287, numbered according to sequence 022048). Any sequence containing any other residue in any of these positions was considered to be non-AOX. In the PTOX run, sequences that PASV identified as having the correct residues in motifs 1 and 2 were annotated as PTOX (motif 1: G157, W158, R160, R161; motif 2: H177, H178, L179, 
A
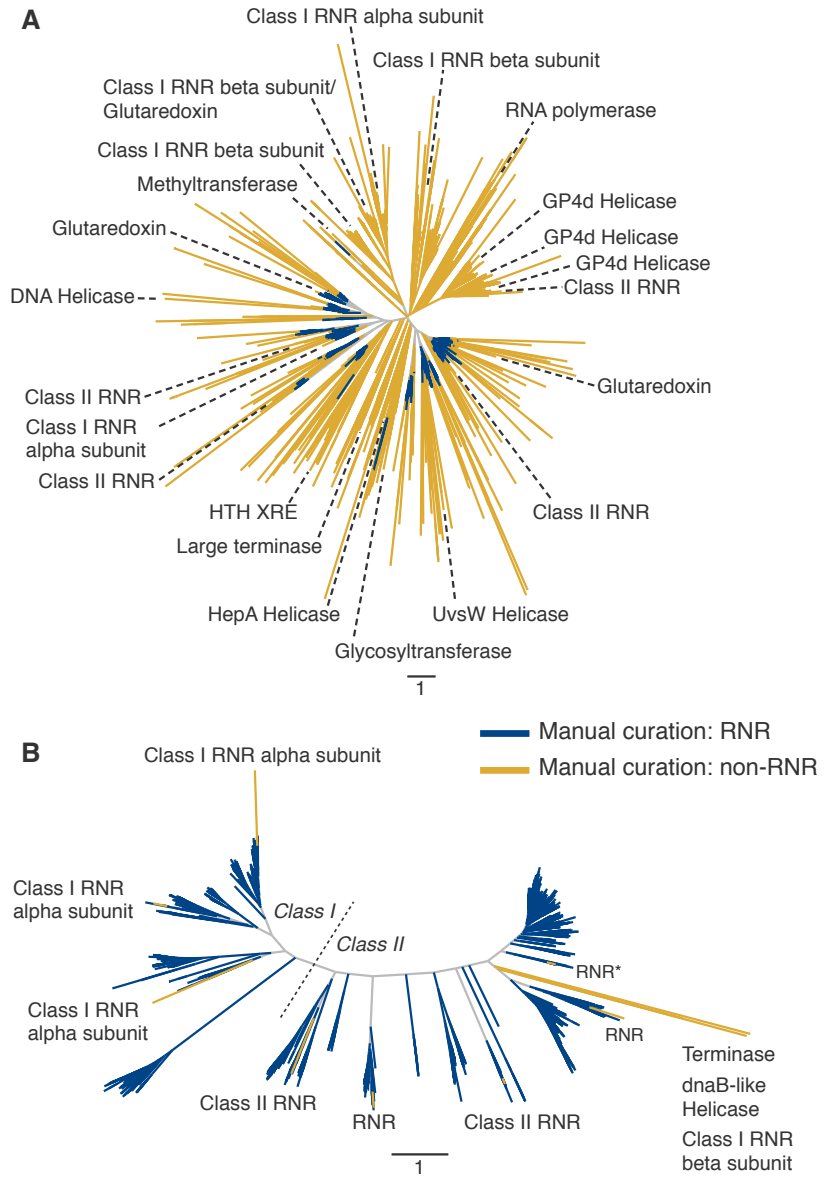

Figure 7. Phylogenetic trees of putative and bonafide GOV RNR sequences. Approximately-maximum likelihood trees of (A) 9,906 putative GOV RNR sequences identified by MMseqs2 using sensitive homology search parameters, and (B) 2,914 PASV validated, bonafide GOV RNR sequences (i.e., sequences with N437, C439, E441, C462, E. coli numbering). In panel B, the dotted line indicates the divide of Class I and Class II RNR sequences. Branch colors correspond to the results of manual curation. Blue branches indicate sequences manually annotated as RNR, whereas yellow branches represent sequences annotated as non-RNR or non-functional RNR sequences. Labelled sequences represent a sampling of sequences with homology to RNR, but manually curated as non-RNR or nonfunctional RNR. Note that some yellow branches in panel B, which were originally annotated as RNRs through manual curation, but having the correct residues according to PASV, were found to have correct RNR annotations according to the NCBI CDD [57]. The branch labeled "RNR*" in panel B indicates 3 branches annotated as RNR by the CDD.

L180, M182, E183, numbered according to sequence AoA061GHF5). Sequences containing different residues in any of these positions were annotated as non-PTOX. When these two annotations were combined, PASV correctly identified all 254 AOX and 82 PTOX peptide sequences and misannotated none.

\section{Discussion}

Homology tools used for collecting gene sequences from databases and metagenomes, such as BLAST [21], HMMER [38], MMseqs2 [39], or PSI-BLAST [40], are sensitive and have the ability to detect remote homology between sequences. While detecting distant homologs is useful, especially when analyzing envi- ronmental metagenomic data, such sensitivity often comes with a price: increased levels of false positive sequences [27]. In the context of viral and microbial ecology, false positives can include non-functional versions of the protein of interest, correctly annotated proteins that do not span a predetermined region of interest, and proteins that share a conserved region or domain with the protein of interest, but are not the desired protein.

Including such false positives in analyses of functional proteins causes a number of problems. False positives interrupt multiple sequence alignments and subsequent phylogenetic analyses, which leads to inaccurate conclusions as to the evolutionary history of a protein $[64,65]$. In ecological studies, inclusion of false positive sequences in marker gene phylogenetic analyses can lead to erroneous identification of microbial or viral populations $[66,37,23]$.

Manual validation of proteins becomes increasingly errorprone and impractical with increasing dataset size. While larger datasets provide the means for deeper exploration of microbial communities and protein diversity and evolution, they also yield more protein sequences for validation. Sensitive homology searches can result in thousands of protein sequences from a single metagenome library, making automatic validation an attractive option.

\section{Using RNRs to test PASV}

Any protein containing conserved residues, whether these are discovered purely through computational methods or are backed by biochemical characterization experiments can be validated using PASV. Ribonucleotide reductase (RNR), an ancient enzyme with well understood structural biochemical features [67] that is often misannotated in sequence databases [36], was an excellent experimental model for testing PASV's ability to validate and partition putative RNR sequences collected from large sequence datasets by homology search. RNRs contain many immutable residues that have been discovered through decades of structural biology research [68]. There is at least one documented case of a gene with high sequence homology to RNR with mutated active sites that has evolved to perform an alternative function [69].

While RNRs are evolutionarily related, perform the same function, and are biochemically conserved, some share only $10-20 \%$ primary sequence similarity, a level below the "twilight zone" of homology search similarity [70, 71, 72]. Searching for RNRs, therefore, requires sensitive homology searches, which can return many false positive sequences. Due to the low level of sequence similarity among RNRs in general, and its many classes and subclasses, RNRs can be difficult to annotate. In one survey of RNRs recovered from GenBank, only $23 \%$ were deemed to be annotated correctly and $16 \%$ had not been annotated as RNRs at all [36]. Given the frequency of misannotation, low sequence homology, presence of immutable residues, and the RNRdb, a large, hand-curated database of bonafide RNR sequences [36], RNR provided an excellent model system for testing PASV. In addition, RNRs are of interest to researchers in many fields, including evolution, biochemistry, cancer research, and viral ecology [62, 18].

Here, we focused on Class I and II RNRs, which are the two most closely related extant RNRs. Class I RNRs are encoded by two genes, one each for the alpha and beta subunits comprising the active protein [67]. The larger alpha subunit is hypothesized to be the direct descendent of Class II RNRs [72], while the beta subunit belongs to the ferritin-like superfamily [73] and bears no homology to either Class I alpha or Class II RNRs. Class I and 
II RNRs require different cofactors for ribonucleotide reduction, so differentiating the classes is crucial for subsequent ecological analyses [18, 23].

PASV was tested using RNRs from two contrasting datasets: the RNRdb [36] and Global Ocean Viromes (GOV) [52]. The majority of RNRs in the RNRdb are from known organisms within large sequence databases (e.g. GenBank, SwissProt, etc.), with relatively few sequences originating from metagenomes. Virus sequences are relatively rare in curated databases as compared to sequences from eukaryotes and bacteria. In fact, viral sequences make up only $2.7 \%$ of the Class I alpha and Class II RNRs in the RNRdb. GOV, in contrast, is an environmental dataset of viral sequences. Thus the RNRdb and GOV represented different challenges for PASV.

\section{Factors influencing PASV accuracy}

The most important factors influencing PASV accuracy surrounded the relative length of reference sequences and the approach used for choosing them. Using full length reference sequences, picking references randomly from a pool of potential sequences rather than based on phylogenies, and using more reference sequences all increased accuracy as measured by true positive and true negative rate. The benefit of using more reference sequences, however, plateaued after ca. 20 sequences in the reference set (Fig. 6), while the computing time required by PASV continued to increase (Additional File 1).

For each phylogenetically-informed reference set generated, a size-matched set of randomly selected RNRs were chosen to act as a control. It is important to note that while the randomly selected sequences are random with respect to their position on the tree, sequences from the RNRdb are biased with respect to class and subclass representation. Therefore, the "random" controls can also be seen as weighted by the composition of the RNRdb.

Alignment software was also a factor, with Clustal Omega generally outperforming MAFFT. However, this advantage was mostly lost when using full-length reference sequences rather than references trimmed to the region of interest. This result may also differ depending on the protein to be aligned, as some datasets are more difficult to align than others [74].

Reference sets representing as much of the known diversity of RNRs as possible (i.e., those taken evenly from across major clades of a phylogenetic tree) were hypothesized to increase PASV accuracy. This hypothesis was built on the idea that including diverse RNRs would prevent large irregularities in the alignments from more divergent query sequences. However, including diverse RNRs had the opposite effect and statistical tests showed that randomly selecting full-length reference sequences resulted in greater accuracy. One explanation for this phenomenon is that accuracy of multiple sequence alignment decreases with increasing sequence heterogeneity $[35,34]$. As a consequence, forcing divergent sequences into the reference sets likely destabilized the alignments and decreased PASV's accuracy.

\section{Using PASV to eliminate bycatch of non-target se- quences}

The GOV dataset provided an alternative experimental model for testing how PASV performed as a post-processing step after a homology search of a metagenomic sequence library. PASV effectively filtered out false-positive bycatch sequences recovered from the environmental metagenomes while searching for the gene of interest, RNR. Of the nearly 10,000 putative RNR sequences identified by MMseqs2, only about one-third were validated as functional RNRs by both PASV and manual curation. The other two-thirds were considered bycatch sequences. Common gene families within the bycatch sequences included RNR Class I beta subunits, thioredoxins, glutaredoxins, polymerases, helicases, and terminases (Additional File 6). Given the sensitivity of MMseqs2 [39], it is likely to find significant hits in sequences only distantly related to RNR or to sequences with domains similar to those occasionally found in RNRs. Some RNR Class I beta subunits are known to contain fused glutaredoxin domains [75]. RNRs may also have regions of remote homology to polymerases, helicases, and terminases as all of these proteins bind DNA. Some RNRs are known to contain zinc-finger domains [76], and at least one of the helicases examined with the CDD contained a zincfinger domain as well (Additional File 6).

Overall, PASV did an excellent job of removing most bycatch sequences (Table 2). Across the 18 reference set experiments that used the full GOV query set, only 187 of 9,906 RNR sequences had PASV predictions that disagreed with manual curation (Additional File 10). In most instances these sequences, annotated as terminases, polymerases, and helicases by NCBI CDD, existed on long branches indicating significant evolutionary distance from true Class I large subunit and Class II RNR sequences (Fig. 7B). Many of the false-positives identified by PASV (those sequences that PASV predicted to be RNRs, but manual curation predicted to be non-RNR) were likely RNR sequences that were missed during manual annotation. This can be attributed to the challenge of manually curating thousands of sequences and the problems inherent when performing large multiple sequence alignments.

\section{Partitioning sequences by key residues}

PASV was conceived as a tool for validating the identity and functionality of protein sequences following homology searches. However, use cases for PASV extend beyond separation of bonafide and bycatch sequences. PASV provides an automated method for applying domain knowledge of a target protein to a large number of sequences. From this domain knowledge, PASV can partition sequences into groups based on structural characteristics that may be linked with protein biochemistry or phylogeny.

PASV was used in such a way to partition Class I alpha and Class II RNRs. While many amino acid residues in the active and allosteric sites of Class I alpha and Class II RNRs are conserved, other residues may be diagnostic of class [23]. Prior work based on protein alignments and phylogenetic trees suggests that the residue in position 438 (E. coli numbering) may be diagnostic of RNR class. Thus, we tested PASV's ability to leverage this domain knowledge by sorting RNRdb sequences into class based on the identity of the residue in position 438 . The function of residue 438 is unknown, but it is known to be conserved and sits within the active finger loop domain that contains the immutable active sites N437, C439, and E441 [77]. The sorting by PASV agreed almost perfectly with the RNRdb class annotations (Table 4), with $>98 \%$ of Class I alpha and Class II sequences correctly identified.

An extension of this use case are peptides that cannot be differentiated by homology searches alone. Alternative oxidase (AOX) and plastid terminal oxidase (PTOX) are membranebound di-iron carboxylate proteins that oxidize a quinol substrate [78]. Although the proteins function within different organelles (AOX functions within the mitochondrial electron transport chain $[79,80]$ while PTOX is a chlororespiration enzyme only found 
within plastids and cyanobacteria [81]), their shared homology and function has led to high levels of misannotation [37]. However, using the amino acid signatures presented previously [37], PASV was able to sort AOX and PTOX proteins from each other with $100 \%$ accuracy. In this way, PASV leverages expert knowledge in an automated fashion.

We have shown that PASV can accurately partition Class I alpha and Class II RNRs using a residue diagnostic of these classes (Table 4), and AOX sequences from PTOX sequences using conserved motifs [37]. Given its success with these two disparate examples, it is likely that PASV could be effectively applied to other gene partitioning tasks as well. For example, a single amino acid mutation at position 762 (E. coli numbering) of motif B of DNA polymerase I ( $\mathrm{Pol} \mathrm{I}$ ) imparts dramatic changes in either the fidelity or efficiency of replication [82]. Subsequent work has hypothesized that Pol I 762 mutations predict the life history characteristics [17] and the genetic composition of the replication module [14] of bacteriophages using Pol I for genome replication. PASV could be used to automatically partition viral Pol I sequences based on the 762 position, providing a means to further test hypothesized connections between Pol I biochemistry and phage life history using large metagenomic datasets. There are many examples of point mutation(s) in bacterial proteins that prevent antibiotics from binding and, thus, inhibit the function of the antibiotic (e.g., K88R in rpsL [83], C117D in murA [84], H526T in rроB [85], Q124K in EF-Tu [86], V246A and V300G in ndh [87]). Such point mutations within a protein would not be readily apparent from homology search alone. Thus PASV could be used for validating and grouping these peptide sequences according to key point mutations following identification via homology search.

\section{Conclusions}

Studies using gene sequences of functional proteins collected from metagenomes for investigating microbial diversity provide new challenges not faced when using genes for stable RNAs like SSU rRNA. These challenges include detecting and preventing false-positive bycatch sequences within datasets, validating key functional residues in proteins of interest, and partitioning peptide sequences into groups or classes. The PASV pipeline provides researchers with a means for addressing these challenges in an automated and highly accurate fashion by combining multiple sequence alignment with expert-curated domain knowledge. The PASV program and source code is freely available under the MIT license and can be found, along with documentation and usage examples, on GitHub: https://github.com/mooreryan/pasv.

\section{Availability of source code and requirements}

- Project name: Protein Active Site Validation (PASV)

- Project home page: https://github.com/mooreryan/pasv

- Operating system(s): Any platform where Ruby and alignment software may be installed, or any platform that supports Docker

- Programming language: Ruby

- Other requirements: Ruby or Docker; alignment software, e.g., Clustal Omega, MAFFT, etc.

- License: MIT

\section{Availability of supporting data and materials}

PASV source code and documentation are available on GitHub at https://github.com/mooreryan/pasv. The PASV Docker image is available on DockerHub at https://hub.docker.com/r/mooreryan/ pasv. Data sets and miscellaneous scripts used in the preparation of the manuscript are available on Zenodo at https://doi. org/10.5281/zenodo.4426410. Additionally, a snapshot of the PASV source code v1.3.0 is available on Zenodo at https://doi.org/10. 5281 /zenodo. 4426410 .

\section{Declarations}

\section{List of abbreviations}

- AOX: alternative oxidase

- BRL: branch length

- CDD: conserved domain database

- GOV: global ocean virome

- IQR: interquartile range

- LOESS: locally estimated scatterplot smoothing

- MSA: multiple sequence alignment

- NCBI: National Center for Biotechnology Information

- PASV: protein active site validation

- PFL: pyruvate formate lyase

- Pol I: DNA polymerase I

- PTOX: plastid terminal oxidase

- RNR: ribonucleotide reductase

- ROI: region of interest

\section{Ethical Approval}

Not applicable.

\section{Consent for publication}

Not applicable.

\section{Competing Interests}

The authors declare that they have no competing interests.

\section{Funding}

This project was supported by the Agriculture and Food Research Initiative grant no. 2012-68003-30155 from the USDA National Institute of Food and Agriculture, the National Science Foundation Advances in Biological Informatics program (award number DBI-1356374), the National Science Foundation Grant No. 1736030, the Established Program to Stimulate Competitive Research (award number OIA-1736030) from the Office of Integrated Activities, and a Doctoral Fellowship provided by University of Delaware in conjunction with the Unidel Foundation. Computational infrastructure support by the University of Delaware Center for Bioinformatics and Computational Biology Core Facility was made possible through funding from the Delaware Biotechnology Institute, and the Delaware INBRE program with a grant from the National Institute of General Medical Sciences (NIGMS P20 GM103446) from the National Institutes of Health and the State of Delaware. The funders had no role in study de- 
sign, data collection and analysis, decision to publish, or preparation of the manuscript. This content is solely the responsibility of the authors and does not necessarily represent the official views of NIH.

\section{Author's Contributions}

- RMM: Conceptualization, Data curation, Formal analysis, Software, Writing - original draft, Writing - review \& editing

- AOH: Conceptualization, Data curation, Writing - review \& editing

- DJN: Conceptualization, Writing - review \& editing

- JC: Conceptualization, Writing - review \& editing

- MC: Conceptualization, Data curation, Visualization

- BDF: Conceptualization, Supervision, Writing - review \& editing

- SWP: Conceptualization, Funding acquisition, Supervision, Writing - review \& editing

- KEW: Conceptualization, Funding acquisition, Supervision, Writing - review \& editing

\section{Acknowledgements}

We would like to acknowledge current and past members of the Viral Ecology and Informatics Lab at the University of Delaware who tested early versions of PASV and provided valuable feedback: Jacob T Dums (ORCID: 0000-0002-6314-4779), Zach Schreiber (ORCID: 0000-0002-6271-2754), and Michael Dahle (ORCID: 0000-0003-0518-3355).

\section{References}

1. Telenti A, Pierce LCT, Biggs WH, di Iulio J, Wong EHM, Fabani $M M$, et al. Deep sequencing of 10,000 human genomes. Proc Natl Acad Sci U S A 2016 Oct;113(42):11901-11906.

2. Lloyd-Price J, Arze C, Ananthakrishnan AN, Schirmer M, Avila-Pacheco J, Poon TW, et al. Multi-omics of the gut microbial ecosystem in inflammatory bowel diseases. Nature 2019 May;569(7758):655-662.

3. Li M, Cao L, Mwimba M, Zhou Y, Li L, Zhou M, et al. Comprehensive mapping of abiotic stress inputs into the soybean circadian clock. Proc Natl Acad Sci U S A 2019 Nov;116(47):23840-23849.

4. Gregory AC, Zayed AA, Conceição-Neto N, Temperton B, Bolduc B, Alberti A, et al. Marine DNA Viral Macro- and Microdiversity from Pole to Pole. Cell 2019 May;177(5):11091123.e14.

5. Koeppel A, Perry EB, Sikorski J, Krizanc D, Warner A, Ward $\mathrm{DM}$, et al. Identifying the fundamental units of bacterial diversity: a paradigm shift to incorporate ecology into bacterial systematics. Proc Natl Acad Sci U S A 2008 Feb;105(7):25042509.

6. Berry MA, White JD, Davis TW, Jain S, Johengen TH, Dick GJ, et al. Are Oligotypes Meaningful Ecological and Phylogenetic Units? A Case Study of Microcystis in Freshwater Lakes. Front Microbiol 2017 Mar;8:365.

7. Mizuno CM, Guyomar C, Roux S, Lavigne R, RodriguezValera F, Sullivan $M B$, et al. Numerous cultivated and uncultivated viruses encode ribosomal proteins. Nat Commun 2019 Feb;10(1):752.

8. Parada AE, Needham DM, Fuhrman JA. Every base mat- ters: assessing small subunit rRNA primers for marine microbiomes with mock communities, time series and global field samples. Environ Microbiol 2016 May;18(5):1403-1414.

9. Bahram M, Anslan S, Hildebrand F, Bork P, Tedersoo L. Newly designed 16S rRNA metabarcoding primers amplify diverse and novel archaeal taxa from the environment. Environ Microbiol Rep 2019 Aug;11(4):487-494.

10. Zhang RY, Zou B, Yan YW, Jeon CO, Li M, Cai M, et al. Design of targeted primers based on 16S rRNA sequences in meta-transcriptomic datasets and identification of a novel taxonomic group in the Asgard archaea. BMC Microbiol 2020 Feb;20(1):25.

11. McLaren MR, Willis AD, Callahan BJ. Consistent and correctable bias in metagenomic sequencing experiments. Elife 2019 Sep; 8.

12. Wooley JC, Ye Y. Metagenomics: Facts and Artifacts, and Computational Challenges*. J Comput Sci Technol 2009 Jan;25(1):71-81.

13. Zhang YZ, Shi M, Holmes EC. Using Metagenomics to Characterize an Expanding Virosphere. Cell 2018 Mar;172(6):11681172.

14. Nasko DJ, Chopyk J, Sakowski EG, Ferrell BD, Polson SW, Wommack KE. Family A DNA Polymerase Phylogeny Uncovers Diversity and Replication Gene Organization in the Virioplankton. Front Microbiol 2018 Dec;9:3053.

15. Sullivan MB. Viromes, not gene markers, for studying double-stranded DNA virus communities. J Virol 2015 Mar;89(5):2459-2461.

16. Nayfach S, Pollard KS. Toward Accurate and Quantitative Comparative Metagenomics. Cell 2016 Aug;166(5):1103-1116.

17. Schmidt HF, Sakowski EG, Williamson SJ, Polson SW, Wommack KE. Shotgun metagenomics indicates novel family A DNA polymerases predominate within marine virioplankton. ISME J 2014 Jan;8(1):103-114.

18. Sakowski EG, Munsell EV, Hyatt M, Kress W, Williamson SJ, Nasko DJ, et al. Ribonucleotide reductases reveal novel viral diversity and predict biological and ecological features of unknown marine viruses. Proc Natl Acad Sci U S A 2014 Nov;111(44):15786-15791.

19. Marine RL, Nasko DJ, Wray J, Polson SW, Wommack KE. Novel chaperonins are prevalent in the virioplankton and demonstrate links to viral biology and ecology. ISME J 2017 Nov;11(11):2479-2491.

20. Chopyk J, Allard S, Nasko DJ, Bui A, Mongodin EF, Sapkota AR. Agricultural Freshwater Pond Supports Diverse and Dynamic Bacterial and Viral Populations. Front Microbiol 2018 Apr;9:3489.

21. Altschul SF, Gish W, Miller W, Myers EW, Lipman DJ. Basic local alignment search tool. J Mol Biol 1990 Oct;215(3):403410.

22. Schnoes AM, Brown SD, Dodevski I, Babbitt PC. Annotation error in public databases: misannotation of molecular function in enzyme superfamilies. PLoS Comput Biol 2009 Dec;5(12):e1000605.

23. Harrison AO, Moore RM, Polson SW, Wommack KE. Reannotation of the Ribonucleotide Reductase in a Cyanophage Reveals Life History Strategies Within the Virioplankton. Front Microbiol 2019 Feb;10:134.

24. Gilks WR, Audit B, De Angelis D, Tsoka S, Ouzounis CA. Modeling the percolation of annotation errors in a database of protein sequences. Bioinformatics 2002 Dec;18(12):16411649.

25. Rinke C, Schwientek P, Sczyrba A, Ivanova NN, Anderson IJ, 
Cheng JF, et al. Insights into the phylogeny and coding potential of microbial dark matter. Nature 2013 Jul;499(7459):431437.

26. Solden L, Lloyd K, Wrighton K. The bright side of microbial dark matter: lessons learned from the uncultivated majority. Curr Opin Microbiol 2016 Jun;31:217-226.

27. Kaushik S, Nair AG, Mutt E, Subramanian HP, Sowdhamini R. Rapid and enhanced remote homology detection by cascading hidden Markov model searches in sequence space. Bioinformatics 2016 Feb;32(3):338-344.

28. Fujimoto MS, Suvorov A, Jensen NO, Clement MJ, Bybee SM. Detecting false positive sequence homology: a machine learning approach. BMC Bioinformatics 2016 Feb;17:101.

29. Fujimoto MS, Suvorov A, Jensen NO, Clement MJ, Snell Q, Bybee SM. The OGCleaner: filtering false-positive homology clusters. Bioinformatics 2017 Jan;33(1):125-127.

30. Fetrow JS. Active Site Profiling to Identify Protein Functional Sites in Sequences and Structures Using the Deacon Active Site Profiler (DASP). Curr Protoc Bioinformatics 2006;14(1):8.10.1-8.10.16.

31. Leuthaeuser JB, Morris JH, Harper AF, Ferrin TE, Babbitt PC, Fetrow JS. DASP3: identification of protein sequences belonging to functionally relevant groups. BMC Bioinformatics 2016 Nov;17(1):458.

32. Harper AF, Leuthaeuser JB, Babbitt PC, Morris JH, Ferrin TE, Poole LB, et al. An Atlas of Peroxiredoxins Created Using an Active Site Profile-Based Approach to Functionally Relevant Clustering of Proteins. PLoS Comput Biol 2017 Feb;13(2):e1005284.

33. Knutson ST, Westwood BM, Leuthaeuser JB, Turner BE, Nguyendac D, Shea G, et al. An approach to functionally relevant clustering of the protein universe: Active site profilebased clustering of protein structures and sequences: Functionally Relevant Clustering of Protein Superfamilies. Protein Sci 2017 Apr;26(4):677-699.

34. Md Mukarram Hossain AS, Blackburne BP, Shah A, Whelan S. Evidence of Statistical Inconsistency of Phylogenetic Methods in the Presence of Multiple Sequence Alignment Uncertainty. Genome Biol Evol 2015 Jul;7(8):2102-2116.

35. Yang K, Zhang L. Performance comparison between k-tuple distance and four model-based distances in phylogenetic tree reconstruction. Nucleic Acids Res 2008 Mar;36(5):e33.

36. Lundin D, Torrents E, Poole AM, Sjöberg BM. RNRdb, a curated database of the universal enzyme family ribonucleotide reductase, reveals a high level of misannotation in sequences deposited to Genbank. BMC Genomics 2009;10(1):589.

37. Nobre T, Campos MD, Lucic-Mercy E, Arnholdt-Schmitt B. Misannotation Awareness: A Tale of Two Gene-Groups. Front Plant Sci 2016 Jun; 7:868.

38. Eddy SR. Accelerated Profile HMM Searches. PLoS Comput Biol 2011 Oct;7(10):e1002195.

39. Steinegger M, Söding J. MMseqs2 enables sensitive protein sequence searching for the analysis of massive data sets. Nat Biotechnol 2017 Nov;35(11):1026-1028.

40. Altschul SF, Madden TL, Schäffer AA, Zhang J, Zhang Z, Miller W, et al. Gapped BLAST and PSI-BLAST: a new generation of protein database search programs. Nucleic Acids Res 1997 Sep;25(17):3389-3402.

41. Sievers F, Wilm A, Dineen D, Gibson TJ, Karplus K, Li W, et al. Fast, scalable generation of high-quality protein multiple sequence alignments using Clustal Omega. Mol Syst Biol 2011 Oct;7:539.

42. Katoh K, Standley DM. MAFFT multiple sequence alignment software version 7: improvements in performance and usability. Mol Biol Evol 2013 Apr;30(4):772-780.

43. Shannon P, Markiel A, Ozier O, Baliga NS, Wang JT, Ramage $D$, et al. Cytoscape: a software environment for integrated models of biomolecular interaction networks. Genome Res 2003 Nov;13(11):2498-2504.

44. Fu L, Niu B, Zhu Z, Wu S, Li W. CD-HIT: accelerated for clustering the next-generation sequencing data. Bioinformatics 2012 Dec;28(23):3150-3152.

45. Kasrayan A, Persson AL, Sahlin M, Sjoberg BM. The conserved active site asparagine in class I ribonucleotide reductase is essential for catalysis. J Biol Chem 2002 Feb;277(8):5749-5755.

46. Mao SS, Yu GX, Chalfoun D, Stubbe J. Characterization of C439SR1, a mutant of Escherichia coli ribonucleotide diphosphate reductase: evidence that $\mathrm{C} 439$ is a residue essential for nucleotide reduction and C439SR1 is a protein possessing novel thioredoxin-like activity. Biochemistry 1992 Oct;31(40):9752-9759.

47. Mao SS, Holler TP, Yu GX, Bollinger JM Jr, Booker S, Johnston MI, et al. A model for the role of multiple cysteine residues involved in ribonucleotide reduction: amazing and still confusing. Biochemistry 1992 Oct;31(40):9733-9743.

48. Persson AL, Eriksson M, Katterle B, Pötsch S, Sahlin M, Sjöberg BM. A New Mechanism-based Radical Intermediate in a Mutant R1 Protein Affecting the Catalytically Essential Glu441 inEscherichia coli Ribonucleotide Reductase. J Biol Chem 1997 Dec;272(50):31533-31541.

49. Letunic I, Bork P. Interactive Tree Of Life (iTOL) v4: recent updates and new developments. Nucleic Acids Res 2019 Jul;47(W1):W256-W259.

50. Price MN, Dehal PS, Arkin AP. FastTree 2-approximately maximum-likelihood trees for large alignments. PLoS One 2010 Mar; 5(3):e9490.

51. Huerta-Cepas J, Serra F, Bork P. ETE 3: Reconstruction, Analysis, and Visualization of Phylogenomic Data. Mol Biol Evol 2016 Jun;33(6):1635-1638.

52. Roux S, Brum JR, Dutilh BE, Sunagawa S, Duhaime MB, Loy $A$, et al. Ecogenomics and potential biogeochemical impacts of globally abundant ocean viruses. Nature 2016 Sep;537(7622):689-693.

53. R Core Team. R: A Language and Environment for Statistical Computing. R Foundation for Statistical Computing, Vienna, Austria; 2020.

54. Wickham H, Averick M, Bryan J, Chang W, McGowan LD, François R, et al. Welcome to the tidyverse. Journal of Open Source Software 2019;4(43):1686.

55. Wickham H. ggplot2: Elegant Graphics for Data Analysis. Springer-Verlag New York; 2016.

56. Moore RM, Harrison AO, McAllister SM, Polson SW, Eric Wommack K. Iroki: automatic customization and visualization of phylogenetic trees. PeerJ $2020 \mathrm{Feb}$;8:e8584.

57. Marchler-Bauer A, Bo Y, Han L, He J, Lanczycki CJ, Lu S, et al. CDD/SPARCLE: functional classification of proteins via subfamily domain architectures. Nucleic Acids Res 2017 Jan;45(D1):D200-D203.

58. Marchler-Bauer A, Lu S, Anderson JB, Chitsaz F, Derbyshire MK, DeWeese-Scott C, et al. CDD: a Conserved Domain Database for the functional annotation of proteins. Nucleic Acids Res 2011 Jan;39(Database issue):D225-9.

59. Kans J. Entrez Direct: E-utilities on the UNIX Command Line. National Center for Biotechnology Information (US); 2020.

60. Harrison AO, Eric Wommack K, editor, Ribonucleotide re- 
ductase genes influence the biology and ecology of marine viruses; 2019.

61. Sawers G. Biochemistry, physiology and molecular biology of glycyl radical enzymes. FEMS Microbiol Rev 1998 Dec;22(5):543-551.

62. Nordlund P, Reichard P. Ribonucleotide reductases. Annu Rev Biochem 2006;75:681-706.

63. Reichard P. From RNA to DNA, why so many ribonucleotide reductases? Science 1993 Jun;260(5115):1773-1777.

64. Ogden TH, Rosenberg MS. Multiple sequence alignment accuracy and phylogenetic inference. Syst Biol 2006 Apr;55(2):314-328.

65. Wong KM, Suchard MA, Huelsenbeck JP. Alignment uncertainty and genomic analysis. Science 2008 Jan;319(5862):473-476.

66. Tripp HJ, Hewson I, Boyarsky S, Stuart JM, Zehr JP. Misannotations of rRNA can now generate $90 \%$ false positive protein matches in metatranscriptomic studies. Nucleic Acids Res 2011 Nov;39(20):8792-8802.

67. Jordan A, Reichard P. Ribonucleotide reductases. Annu Rev Biochem 1998;67:71-98.

68. Kolberg M, Strand KR, Graff P, Andersson KK. Structure, function, and mechanism of ribonucleotide reductases. Biochim Biophys Acta 2004 Jun;1699(1-2):1-34.

69. Lembo D, Donalisio M, Hofer A, Cornaglia M, Brune W, Koszinowski $\mathrm{U}$, et al. The ribonucleotide reductase R1 homolog of murine cytomegalovirus is not a functional enzyme subunit but is required for pathogenesis. J Virol 2004 Apr;78(8):4278-4288.

70. Rost B. Twilight zone of protein sequence alignments. Protein Eng 1999 Feb;12(2):85-94.

71. Torrents E. Ribonucleotide reductases: essential enzymes for bacterial life. Front Cell Infect Microbiol 2014 Apr;4:52.

72. Lundin D, Berggren G, Logan DT, Sjöberg BM. The origin and evolution of ribonucleotide reduction. Life 2015 Feb;5(1):604-636.

73. Andrews SC. The Ferritin-like superfamily: Evolution of the biological iron storeman from a rubrerythrin-like ancestor. Biochim Biophys Acta 2010 Aug;1800(8):691-705.

74. Wang LS, Leebens-Mack J, Kerr Wall P, Beckmann K, dePamphilis CW, Warnow T. The impact of multiple protein sequence alignment on phylogenetic estimation. IEEE/ACM Trans Comput Biol Bioinform 2011 Jul;8(4):1108-1119.

75. Rozman Grinberg I, Lundin D, Sahlin M, Crona M, Berggren G, Hofer A, et al. A glutaredoxin domain fused to the radicalgenerating subunit of ribonucleotide reductase (RNR) functions as an efficient RNR reductant. J Biol Chem 2018 Oct;293(41):15889-15900.

76. Loderer C, Jonna VR, Crona M, Rozman Grinberg I, Sahlin $\mathrm{M}$, Hofer $\mathrm{A}$, et al. A unique cysteine-rich zinc finger domain present in a majority of class II ribonucleotide reductases mediates catalytic turnover. J Biol Chem 2017 Nov;292(46):19044-19054.

77. Eklund $\mathrm{H}$, Uhlin U, Färnegårdh $\mathrm{M}$, Logan DT, Nordlund $\mathrm{P}$. Structure and function of the radical enzyme ribonucleotide reductase. Prog Biophys Mol Biol 2001 Nov;77(3):177-268.

78. Berthold DA, Stenmark P. Membrane-bound diiron carboxylate proteins. Annu Rev Plant Biol 2003;54:497-517.

79. Affourtit C, Albury MS, Crichton PG, Moore AL. Exploring the molecular nature of alternative oxidase regulation and catalysis. FEBS Lett 2002 Jan;510(3):121-126.

80. McDonald A, Vanlerberghe G. Branched mitochondrial electron transport in the Animalia: presence of alterna- tive oxidase in several animal phyla. IUBMB Life 2004 Jun;56(6):333-341.

81. Carol P, Kuntz M. A plastid terminal oxidase comes to light: implications for carotenoid biosynthesis and chlororespiration. Trends Plant Sci 2001 Jan;6(1):31-36.

82. Tabor $\mathrm{S}$, Richardson CC. A single residue in DNA polymerases of the Escherichia coli DNA polymerase I family is critical for distinguishing between deoxy- and dideoxyribonucleotides. Proc Natl Acad Sci U S A 1995 Jul;92(14):6339-6343.

83. Ballif $M$, Harino $P$, Ley $S$, Coscolla $M$, Niemann S, Carter $\mathrm{R}$, et al. Drug resistance-conferring mutations in Mycobacterium tuberculosis from Madang, Papua New Guinea. BMC Microbiol 2012 Sep;12:191.

84. De Smet KAL, Kempsell KE, Gallagher A, Duncan K, Young DB. Alteration of a single amino acid residue reverses fosfomycin resistance of recombinant MurA from Mycobacterium tuberculosis The EMBL accession number for the sequence in this paper is X96711. Microbiology 1999 Nov;145(11):3177-3184.

85. Sajduda A, Brzostek A, Poplawska M, Augustynowicz-Kopec E, Zwolska Z, Niemann S, et al. Molecular characterization of rifampin- and isoniazid-resistant Mycobacterium tuberculosis strains isolated in Poland. J Clin Microbiol 2004 Jun;42(6):2425-2431.

86. Zuurmond AM, Olsthoorn-Tieleman LN, Martien de Graaf J, Parmeggiani A, Kraal B. Mutant EF-Tu species reveal novel features of the enacyloxin IIa inhibition mechanism on the ribosome. J Mol Biol 1999 Dec;294(3):627-637.

87. Vilchèze $\mathrm{C}$, Weisbrod TR, Chen $\mathrm{B}$, Kremer L, Hazbón $\mathrm{MH}$, Wang F, et al. Altered NADH/NAD+ ratio mediates coresistance to isoniazid and ethionamide in mycobacteria. Antimicrob Agents Chemother 2005 Feb;49(2):708-720. 